\title{
Tree Trunk Inspections Using a Polarimetric GPR System
}

\author{
Lilong Zou*a, Livia Lantini ${ }^{\mathrm{a}}$, Fabio Tosti ${ }^{\mathrm{a}}$, Amir M. Alani ${ }^{\mathrm{a}}$ \\ aSchool of Comp. and Engin., Univ. of West London/London, St Mary’s Road, UK, W5 5RF
}

\begin{abstract}
In this work, a novel signal processing framework for polarimetric GPR measurements is presented for inspection of tree trunks decay. The framework combines a polarimetric noise filter and an arc-shaped diffraction imaging algorithm. The polarimetric noise filter can increase the signal-to-noise ratio (SNR) of B-scans caused by the bark and the high-loss propriety of the tree trunk based on a 3D Pauli feature vector of the Bragg scattering theory. The arc-shaped diffraction stacking and an imaging aperture are then designed to suppress the effects of the irregular shape of the tree trunk on the signal. The proposed detection scheme is successfully validated with real tree trunk measurements. The viability of the proposed processing framework is demonstrated by the high consistency between the results and the real-truth trunk cross-sections.
\end{abstract}

Keywords: non-destructive testing (NDT), ground penetrating radar (GPR), tree trunk, polarimetric radar, Bragg Scattering

\section{INTRODUCTION}

In recent years, an increasing number of pests and diseases have been causing damage to trees in many European countries, particularly in the UK. Entire forests and woodlands, which threaten the survival of certain tree species in the UK, are affected by multiple forms of virus, also known as emerging infectious diseases (EIDs). EIDs are increasingly being recognised as a global threat to human progress, such as food security and global warming. With the advancement of modern science, the threat of plant diseases has not yet diminished. In fact, EIDs have become more severe due to trade and transport factors, as well as the microbial adaptation to new ecosystems as a result of climate changes. The impact of the spread of EIDs is now dramatic, as these diseases are increasing and could lead to the complete extinction of certain tree species. This causes trees to become starved and even die. As predictive models and small-scale studies will hardly be capable to provide fully comprehensive predictions of future trends of disease spread, intensive monitoring and surveillance will become increasingly necessary protocols to develop in the coming years.

Therefore, controlling and reducing EIDs is a complex and important task. In addition to controlling its spread, a fast detection of tree diseases is also required to provide prompt remedial actions. However, early signs of tree disease usually appear in the inner centre of the tree. Hence, they are difficult to detect from the outer surface of the tree. Ground Penetrating Radar (GPR) is becoming increasingly common in environmental engineering as a recognised nondestructive testing (NDT) tool [1]-[4]. GPR is a real-time NDT technique that uses high-frequency radio waves to generate high-resolution data with a fast data collection time [5]. The technique uses electromagnetic waves, which propagate at a specific speed determined by the dielectric constant of the material [6][7]. However, issues exist with using traditional ground-penetrating radar calculations for nondestructive tree inspection. First, live trees usually have a high moisture content, which is not conducive to the propagation of high-frequency electromagnetic waves within the trunk of the tree [8]. Second, the choice of a lower frequency system may result in higher penetration depths. However, it can be unpractical due to system size limitations. Finally, unlike surface conditions, the irregular shape of tree trunks is not straightforward for conventional data processing [9].

The motivation of this study is to present new signal processing algorithms for non-destructive tree trunk inspection using a dual-polarised GPR system. Polarisation is a property of electromagnetic waves that describes the direction of the electric field vector and can be used to characterise target properties by polarisation radar. The processing framework is presented based on a 3D Pauli feature vector of Bragg Scattering and an arc-shaped diffraction imaging algorithm. The first method is utilised to eliminate noise created by the bark and high loss characteristics of trunk. The arc diffraction imaging method and imaging aperture are also designed to reduce the effect of the uneven shape of tree trunk. Measurements of real tree trunks were used to properly validate the proposed data processing methodology.

*lilong.zou@uwl.c.uk 


\section{THEORETICAL FRAMEWORK}

The processing pipeline of this study consists of three sequential stages. A pre-processing algorithm for time correction and signal noise filtering is initially applied to increase the overall signal-to-clutter ration. Subsequently, the polarimetric noise filter is employed to suppress the noise created by the bark and the trunk high loss characteristics. In the last stage, an arc-diffraction imaging method is designed to reduce the effect of the irregular shape of the tree trunk.

\subsection{Pre-processing Algorithm}

The data pre-processing procedures including time-zero adjustment and background removal were performed on the acquired raw data. To adjust all the traces to the zero-time position before other processing methods, zero-time correction must be carried out first. In GPR signals, the cross-coupling between the transmitter and the receiver as well as the ringing noise and multiple reflections can mask the less dominant reflections from the bark. To mitigate such clutter, background removal (clutter rejection) method should be applied.

\subsection{Polarimetric Noise Filter}

Polarimetric noise filter is applied to improve the quality of the information in the radargram by removing different types of noise. A general formulation for polarimetric radar is presented in full details by Cloude and Papathanassiou [10]. Fully polarimetric radar systems measure a $2 \times 2$ complex scattering matrix for each channel dataset. Through vectorisation of the scattering matrix, a coherent scattering vector $k$ can be extracted to generalise the spatial coherence [11]. For a monostatic backscattering case, the reciprocity constrains the Sinclair scattering matrix to be symmetrical, that is, $S_{H V}=S_{V H}$. Thus, the 4-D polarimetric target vectors reduce to 3-D polarimetric target vectors. Using the Pauli basis matrices, the scattering vector for the monostatic case can be found as [12]

$$
k=\left[\begin{array}{c}
S_{H H}+S_{V V} \\
S_{H H}-S_{V V} \\
2 S_{H V}
\end{array}\right]
$$

For the Bragg scattering, one may assume that there is only one dominant eigenvector (Depolarisation is negligible) and the eigenvector is given by:

$$
k=\left[\begin{array}{c}
S_{H H}+S_{V V} \\
S_{H H}-S_{V V} \\
0
\end{array}\right]
$$

The “Aladdin” dual-polarised GPR system used in this study is manufactured by IDS GeoRadar (Part of Hexagon) (Figure 1). This system is equipped with two $2 \mathrm{GHz}$ antennas polarised perpendicular each to one another, so that $\mathrm{HH}$ and VV channels polarimetric data can be acquired at the same time. After preprocessing the dual channel raw data, the 3D Pauli feature vector is performed. In the radar profile composed by the first component $\left(S_{H H}+S_{V V}\right)$, the random or decorrelated noise is much compressed. Conversely, in the radar profile composed by the second component $\left(S_{H H}-S_{V V}\right)$, the coherent noise generated by the system, bulk multi-reflection are much reduced. Then the polarimetric noise filter is applied to $\left(S_{H H}+S_{V V}\right)^{2}-\left|\left(S_{H H}-S_{V V}\right)^{2}\right|$ radar profile to increase the SNR of the data.

\subsection{Arc-diffraction Imaging}

To reduce the effect of the irregular shape of tree trunk, the diffraction image is transformed to cylindrical coordinate system. The proposed algorithm requires knowledge of the azimuth angle, the radius and the altitude. These two-way travel time should be computed for each grid point as they are potential target locations. Meanwhile, the radar response at the reverse arc corresponding to the calculation point should not be used in the diffraction stacking calculation at this point. In the reconstructed image, the high value part can be associated with the presence of decays or anomalies in the tree trunk. Then, the depth location of decay can be estimated from this reconstructed image.

\section{EXPERIMENT RESULTS}

The proposed methodology is tested on real measurements collected at the Kensington Gardens - The Royal Parks - in London, UK. The investigated tree is shown in Figure 2 (left). The time-step used for data collection purposes are dt = 
$6.25 \times 10^{-11} \mathrm{~s}$ and $1 \mathrm{~cm}$, respectively. Circular scans were taken every $5 \mathrm{~cm}$ along the tree main longitudinal axis and parallel to the ground. The total scanned area was $1.45 \mathrm{~m}$ long and was made up of 30 circular scans in a row. The inspected area is therefore semi-cylindrical in shape, with a varying circumference due to the bark shape irregularities. Using the measuring-wheel device attached to the antenna, the circumference of each portion was correctly calculated and then incorporated into the detection technique. The raw GPR profiles from an individual circular scan of $\mathrm{HH}$ and VV channels are shown in Figure 2(b-c).

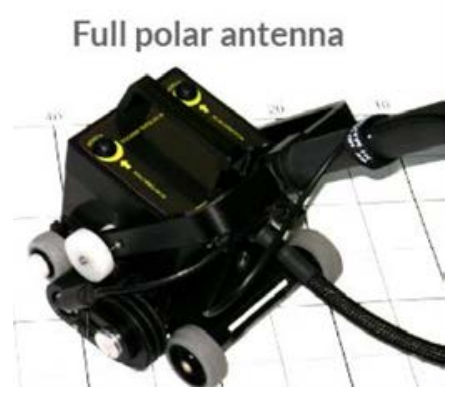

Figure 1. The Aladdin dual-polarised GPR system - manufactured by IDS GeoRadar (Part of Hexagon) - used in this study.

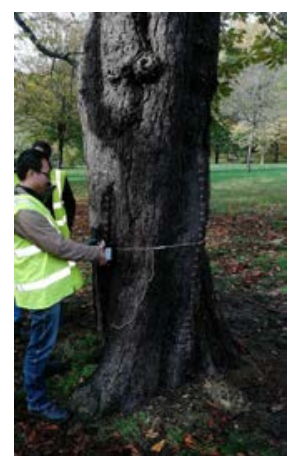

(a)

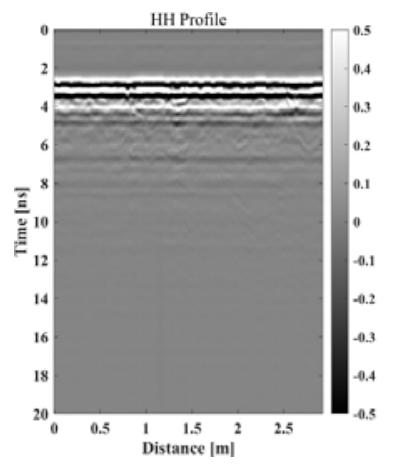

(b)

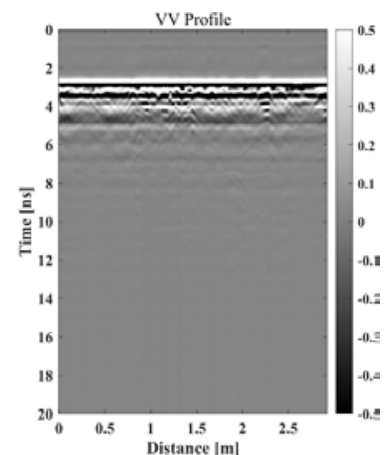

(c)

Figure 2. The tree under investigation (a) and the polarimetric radar profiles (HH (b) and VV (c)).

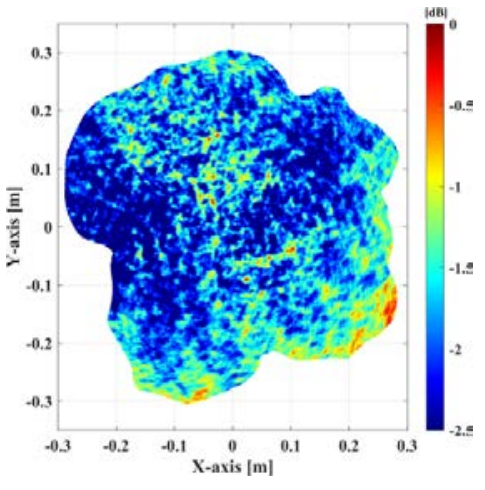

(a)

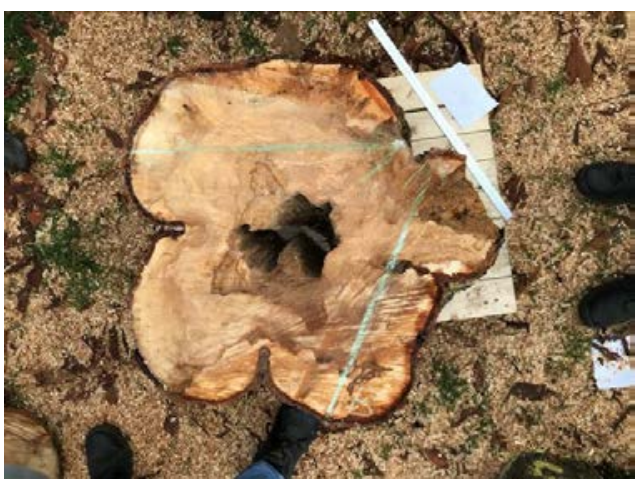

(b)

Figure 3. Reconstructed image of tree trunk by the proposed approach (a) and the corresponding real section of the trunk (b).

After completion of measurements, the tree was torn down and chopped into several slices to reveal its underlying structure (see Figures 3(b) and 4(b)). A significant deterioration spreading along the major axis of the trunk can be clearly seen in Figures 4 and 5. Figures 3(b) and 4(b) illustrate the reconstructed image using the methodology described in this paper. Clear evidence of a major feature at the centre of the trunk are observed. Moreover, a hollow exists inside this major feature. From the reconstructed image, we could clearly see that the results agree with the actual structure of 
the tree. The shape and the size of the decay are adequately reconstructed. This suggests that GPR coupled with the proposed methodology can accurately detect hidden decay in an efficient and practical manner.

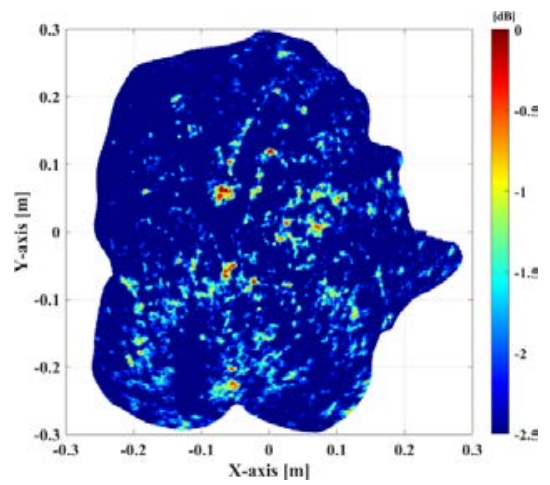

(a)

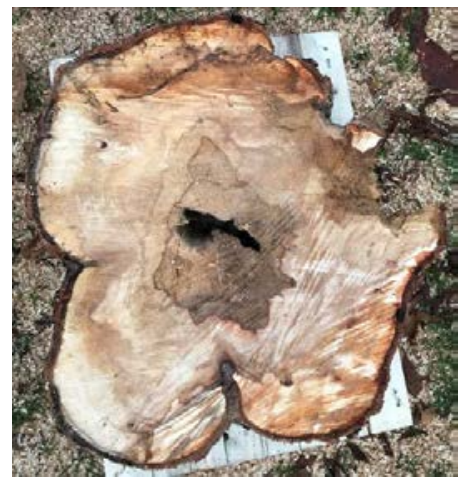

(b)

Figure 4. Reconstructed image of tree trunk by the proposed approach (a) and the corresponding real section of the trunk (b).

\section{CONCLUSION AND FUTURE PROSPECTS}

In this paper, a novel processing framework for polarimetric GPR measurements is presented for inspection of tree trunks decay. The polarimetric noise filter based on the 3D Pauli feature vector of the Bragg Scattering can increase the signal-to-noise ratio (SNR) of B-scans caused by the bark and the high-loss propriety of the tree trunk. The arc-shaped diffraction stacking and an imaging aperture are then designed to suppress the effects of the irregular shape of the tree trunk on the signal. Measurements of real tree trunks were used to properly validate the proposed data processing methodology. The viability of the proposed processing framework is demonstrated by the high consistency between the results and the real trunk cross-sections. Future research could task itself to create realistic non-destructive pseudo-3D model of the trunk by combination of 2D tomographic images.

\section{REFERENCES}

[1] Alani, A.M.; Soldovieri, F.; Catapano, I.; Giannakis, I.; Gennarelli, G.; Lantini, L.; Ludeno, G.; Tosti, F., “The Use of Ground Penetrating Radar and Microwave Tomography for the Detection of Decay and Cavities in Tree Trunks,” Remote Sens. Papers 11, 2073 (2019).

[2] Benedetto, A.; Tosti, F.; Ortuani, B.; Giudici, M.; Mele, M., "Soil moisture mapping using GPR for pavement applications,” Proc. IWAGPR, 1-5 (2013).

[3] Themistocleous, K.; Neocleous, K.; Pilakoutas, K.; Hadjimitsis, D.G. , "Damage assessment using advanced nonintrusive inspection methods: Integration of space, UAV, GPR, and field spectroscopy,” Proc. RSCy2014, 92291O1-92291O-5 (2014).

[4] Zou, L.; Wang, Y.; Giannakis, I.; Tosti, F.; Alani, A.M.; Sato, M., "Mapping and Assessment of Tree Roots Using Ground Penetrating Radar with Low-Cost GPS,” Remote Sens. Papers 12, 1300 (2020).

[5] Daniels, D. J., [Ground Penetrating Radar], 2nd ed. London, U.K.: Institution of 563 Engineering and Technology, (2004).

[6] Zou, L., Yi, L., and Sato, M., "On the Use of Lateral Wave for the Interlayer Debonding Detecting in an Asphalt Airport Pavement Using a Multistatic GPR System,” IEEE Trans. Geo. Remote Sens. Papers 58(6), 4215-4224 (2020).

[7] Zou, L., Kikuta, K., Alani, A.M., and Sato, M., "Study on Wavelet Entropy for Airport Pavement Inspection using a Multi-Static GPR System,” Geophysics Papers 86(3), WB69-WB79, (2021).

[8] Giannakis, I.; Tosti, F.; Lantini, L.; Alani, A. M., "Diagnosing Emerging Infectious Diseases of Trees Using Ground Penetrating Radar,” IEEE Trans. Geo. Remote Sens. Papers, 58, 1146-1155 (2020).

[9] Giannakis, I.; Tosti, F.; Lantini, L.; Alani, A. M., "Health Monitoring of Tree Trunks Using Ground Penetrating Radar," IEEE Trans. Geo. Remote Sens. Papers, 57, 8317-8326 (2019).

[10] Van Zyl, J. and Kim, Y., [Synthetic Aperture Radar Polarimetric], NJ, Hoboken: Wiley, (2011).

[11] Mattia, F., Le Toan, T., Souyris, J. C., Carolis, G. D., and Floury, N., "The effect of surface roughness on multifrequency polarimetric SAR data,” IEEE Trans. Geo. Remote Sens. Papers 35(4), 954-966 (1997).

[12] Lee, J. and Pottier, E., [Polarimetric Imaging: From Basics to Applications], FL, Boca Raton: CRC Press, (2009). 